\title{
Association of Hyponatremia and Clinical Prognosis in Neuro Critically Ill Patients
}

\author{
Changmin $\mathrm{Ha}^{1}$, Jeong-Am Ryu ${ }^{1,2}$ \\ ${ }^{1}$ Department of Neurosurgery, Samsung Medical Center, Sungkyunkwan University School of Medicine, Seoul, Korea \\ ${ }^{2}$ Department of Critical Care Medicine, Samsung Medical Center, Sungkyunkwan University School of Medicine, Seoul, Korea
}

Received: April 13, 2021

Accepted: April 25, 2021

Corresponding Author: Jeong-Am Ryu, M.D., Ph.D. Department of Critical Care Medicine and Department of Neurosurgery, Samsung Medical Center, Sungkyunkwan University School of Medicine, 81 Irwon-ro, Gangnam-gu, Seoul 06351, Korea Tel: 82-2-3410-6399

Fax: 82-2-2148-7088

E-mail:lamyud.ryu@samsung.com

\section{Objective}

To investigate the impact of hyponatremia severity on in-hospital mortality of neurocritically ill patients.

\section{Methods}

This was a retrospective, observational study of patients who were admitted at a tertiary university hospital, Seoul, Republic of Korea, neurosurgical intensive care unit (ICU) from January 2013 to December 2019. We included neurocritically ill patients whose serum sodium concentrations were obtained during the ICU admission. The primary endpoint was in-hospital mortality.

Results

A total of 903 patients were included in the final analysis. Hyponatremia was detected in 359 (39.8\%) patients. There was no significant difference in in-hospital mortality between the no hyponatremia group and the hyponatremia group $(8.8 \%$ vs. 9.7\%, $p=0.724)$. However, the length of ICU stay was longer in the hyponatremia group than no hyponatremia group $(282.3 \pm 295.9 \mathrm{hr}$ vs. $141.8 \pm 89.3 \mathrm{hr}, p<0.001$ ). In the multivariable analysis, severe hyponatremia (adjusted odds ratio [OR]: 5.41, 95\% confidence interval [CI]: 1.23 - 20.54), malignancy (adjusted OR: 3.45, 95\% CI: 1.29 - 9.72), continuous renal replacement therapy (adjusted OR: 6.15, 95\% CI: 1.65 23.38), invasive intracranial pressure monitoring (adjusted OR: 0.39, 95\% CI: $0.17-0.83$ ) and Glasgow Coma Scale on ICU admission (adjusted OR: 0.6, 95\% CI: 0.52 - 0.69) were significantly associated with in-hospital mortality.

\section{Conclusion}

Based on our findings, mild to moderate hyponatremia were not associated with clinical outcomes. However, severe hyponatremia was significantly associated with in-hospital mortality. Therefore, adequate and timely regulation of serum sodium level is important for favorable prognosis and survival of neurocritically ill patients.

Keywords: Hyponatremia; Prognosis; Neurosurgery; Intensive care unit 


\section{INTRODUCTION}

Hyponatremia is a common electrolyte abnormality in hospitalized patients ${ }^{6,9,13,17)}$. It could be mild with nausea and vomiting or severe with lethargy, seizures, and loss of consciousness ${ }^{14}$. In the critically ill, especially neurocritically ill patients, hyponatremia is also a common electrolyte disorder and is associated with clinical prognosis ${ }^{13,14)}$. Hyponatremia may induce neurological complications including cerebral edema, increased intracranial pressure (ICP), seizures, altered mentality, and brain death due to exacerbation of brain edema ${ }^{6}$. In addition, neurocritically ill patients may be more vulnerable to hyponatremia and may develop clinical symptoms in the absence of severe hyponatremia ${ }^{6,16)}$. Therefore, inadequate or delayed hyponatremia treatment may lead to significant morbidity and mortality ${ }^{13)}$ in neurocritically ill patients. However, the number of studies that have reported the correlation between hyponatremia severity and the clinical outcomes of neurocritically ill patients is limited. In this study, we aimed to evaluate the impact of hyponatremia severity on in-hospital mortality of neurocritically ill patients.

\section{MATERIALS AND METHODS}

\section{Study population and design}

This was a retrospective, single-center, observational study of patients who were admitted at the Samsung Medical Center, Seoul, Republic of Korea, neurosurgical intensive care unit (ICU) from January 2013 to December 2019. This study was approved by the Institutional Review Board (IRB) of Samsung Medical Center (IRB approval number: SMC 2020-09-082). Patients' records were reviewed and analyzed according to the Declaration of Helsinki. The requirement of informed consent was waived by the IRB due to its retrospective nature. We included neurocritically ill patients and those who were on neurosurgical postoperative management, in cluding brain tumor, subarachnoid hemorrhage, cerebral vascular surgery, intracerebral hemorrhage, cerebral infarction, traumatic brain injury, infection of the central nervous system. In addition, the patients should have been hospitalized in the ICU for more than 3 days or died within 3 days after ICU admission, and their serum sodium concentrations should have been obtained during the ICU admission. We excluded patients whose hypernatremia was above $145 \mathrm{mEq} / \mathrm{L}$ during their ICU stay, who had insufficient medical records, who had a 'do not resuscitation' order, who were admitted to departments other than neurosurgery, and who were transferred to other hospitals or with unknown prognosis (Fig. 1).

Definitions and outcomes In this study, the baseline characteristics such as comorbidities, behavioral risk factors, ICU management, and laboratory data were collected retrospectively using Clinical Data Warehouse. Our center constructed a "Clinical Data Warehouse Darwin-C" designed for investigators to search and retrieve de-identified medical records from electronic archives. The serum sodium levels were obtained at least once every day from all neurosurgical patients. Hyponatremia was defined as serum sodi$\mathrm{um}<135 \mathrm{mmol} / \mathrm{L}^{5)}$. In addition, to help determine the association between the clinical outcomes and severity of hyponatremia, the patients were categorized into four subgroups: no hyponatremia ( $\geq 135 \mathrm{mEq} / \mathrm{L})$, mild hyponatremia $(130-134 \mathrm{mEq} / \mathrm{L})$, moderate hyponatremia $(125-129 \mathrm{mEq} / \mathrm{L})$, and severe hypernatremia $(<125 \mathrm{mEq} / \mathrm{L})^{5)}$. In this study, the primary endpoint was in-hospital mortality.

\section{Statistical analyses}

All data are presented as mean \pm standard deviation for contin-

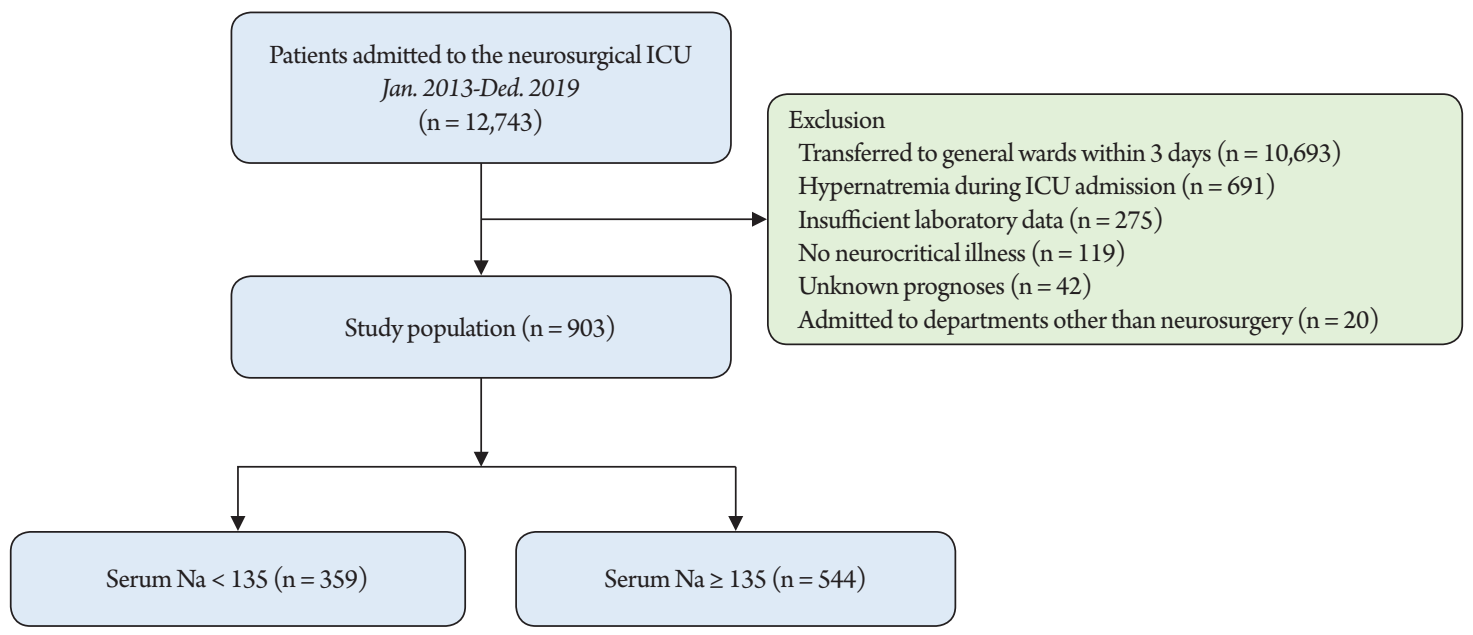

Fig. 1. Study flow chart. ICU: Intensive care unit. 
uous variables or frequencies and proportions for categorical variables. Data were compared using Student's $t$-test for continuous variables and Chi-square test or Fisher's exact test for categorical variables. A stepwise multiple logistic regression was performed to obtain a statistically meaningful predictor of in-hospital mortality. All tests were two-sided and $p$ values of less than 0.05 were considered statistically significant. All statistical analyses were performed with R Statistical Software version 4.0.2 (R Foundation for Statistical Computing, Vienna, Austria).

\section{RESULTS}

\section{Baseline characteristics}

A total of 12,743 patients were admitted to the neurosurgical
ICU during the study period, among them 903 patients were included in the final analysis. The mean age of all the patients was $44.9 \pm 24.4$ years and $54.0 \%$ of patients were male. Hyponatremia was detected in 359 (39.8\%) patients. Malignancy (66.0\%) and hypertension $(37.1 \%)$ were the most common comorbidities, while brain tumors (52.2\%) and intracerebral hemorrhage (12.7\%) were the most common reasons for ICU admission (Table 1).

\section{Clinical outcomes of the overall study population}

There was no significant difference in in-hospital mortality between the no hyponatremia group and the hyponatremia group ( $8.8 \%$ vs. $9.7 \%, p=0.724$ ). However, the length of ICU stay was longer in the hyponatremia group than no hyponatremia group $(282.3 \pm 295.9 \mathrm{hr}$ vs. $141.8 \pm 89.3 \mathrm{hr}, p<0.001)$. In addition,

Table 1. Baseline characteristics

\begin{tabular}{|c|c|c|c|}
\hline Variable & Normal $(n=544)$ & Hyponatremia $(\mathrm{n}=359)$ & $p$ value \\
\hline \multicolumn{4}{|l|}{ Patient demographics } \\
\hline Age (year) & $43.4 \pm 24.1$ & $47.2 \pm 24.7$ & 0.021 \\
\hline Sex, male & $276(50.7)$ & $212(59.1)$ & 0.017 \\
\hline \multicolumn{4}{|l|}{ Comorbidities } \\
\hline Malignancy & $332(61.0)$ & $264(73.5)$ & $<0.001$ \\
\hline Hypertension & $147(27.0)$ & $112(31.2)$ & 0.200 \\
\hline Chronic kidney disease & $19(3.5)$ & $31(8.6)$ & 0.002 \\
\hline Cardiovascular disease & $19(3.5)$ & $13(3.6)$ & 0.999 \\
\hline Chronic liver disease & $13(2.4)$ & $12(3.3)$ & 0.518 \\
\hline \multicolumn{4}{|l|}{ Behavioral risk factors } \\
\hline Current alcohol consumption & $100(18.4)$ & $68(18.9)$ & 0.901 \\
\hline Intracerebral hemorrhage & $69(12.7)$ & $46(12.8)$ & \\
\hline Vascular surgery & $70(12.9)$ & $34(9.5)$ & \\
\hline Traumatic brain injury & $54(9.9)$ & $35(9.7)$ & \\
\hline Subarachnoid hemorrhage & $52(9.6)$ & $34(9.5)$ & \\
\hline Central nervous system infection & $8(1.5)$ & $14(3.9)$ & \\
\hline Cerebral infarction & $15(2.8)$ & $1(0.3)$ & \\
\hline APACHE II score on ICU admission & $5.0 \pm 5.0$ & $5.9 \pm 5.5$ & 0.006 \\
\hline Glasgow Coma Scale on ICU admission & $13.1 \pm 2.4$ & $13.0 \pm 2.3$ & 0.696 \\
\hline \multicolumn{4}{|l|}{ ICU management } \\
\hline Mechanical ventilation & $182(33.5)$ & $162(45.1)$ & 0.001 \\
\hline
\end{tabular}

Data are presented as numbers (\%) or means \pm standard deviations.

APACHE: Acute Physiology and Chronic Health Evaluation, ICP: intracranial pressure, ICU: intensive care unit, ICP: intracranial pressure.

"Some patients received more than one hyperosmolar agent. 
there were no significant differences in the clinical outcome according to the hyponatremia severity-based groups except hospital length of stay $(p<0.001)$ (Table 2).

In the multivariable analysis, severe hyponatremia (adjusted odds ratio [OR]: 5.41, 95\% confidence interval [CI]: 1.23 - 20.54), malignancy (adjusted OR: 3.45, 95\% CI: 1.29 - 9.72), continuous renal replacement therapy (adjusted OR: 6.15, 95\% CI: 1.65 - 23.38), invasive ICP monitoring (adjusted OR: 0.39, 95\% CI: 0.17 - 0.83) and Glasgow Coma Scale on ICU admission (adjusted OR: 0.6, $95 \%$ CI: $0.52-0.69)$ were significantly associated with in-hospital mortality (Table 3).

In the survival rate analysis, the patients with severe hyponatremia had the worst survival rate among the overall patients. However, there were no significant differences in the mortality rates of patients according to the severity of hyponatremia ( $p=0.430$, Fig. 2 ).

\section{DISCUSSION}

In this study, we investigated the impact of hyponatremia on in-hospital mortality of neurocritically ill patients. Major findings of this study were as follows. First, based on our findings, approximately one-third of the neurocritically ill patients had hyponatremia during their ICU stay. However, there was no significant difference in in-hospital mortality between the no hyponatremia group and the hyponatremia group. There were also no significant differences in the clinical outcome among the hyponatremia severity based. On the other hand, the length of ICU stay was longer in the hyponatremia group than in the no hyponatremia group. Although hyponatremia did not show significant association with clinical outcomes in the univariable analysis, severe hyponatremia showed significant association with mortality in multivariable analysis. In addition, the multivariable analysis revealed that severe hyponatremia, malignancy, continuous renal replacement therapy, invasive ICP monitoring, and Glasgow Coma Scale on ICU admission were significantly associated with in-hospital mortality.

Hyponatremia is the most common electrolyte abnormality in hospitalized patients ${ }^{3)}$ and especially the neurocritically ill patients $^{9)}$; previous studies reported hyponatremia in up to $38 \%$ of critically ill patients and up to $50 \%$ of neurosurgical patients $^{2,7,9,16,19)}$. Although the pathophysiology of hyponatremia in neurocritically ill patients is multifactorial, cerebral salt wasting syndrome and syndrome of inappropriate antidiuretic are the most common causes of hyponatremia in these patients ${ }^{6,9,13)}$. Hyponatremia has been associated with the clinical progress of neurocritically ill patients ${ }^{9,13,15)}$. Acute hyponatremia can cause brain edema and rapid neurological deterioration by increasing the osmotic gradient between the cerebral interstitial tissue and serum ${ }^{9}$. If prolonged, hyponatremia even in mild cases can lead to increased in-

Table 2. Clinical outcome according to the severity of hyponatremia

\begin{tabular}{lccrrr}
\hline Variable & No hyponatremia $(\mathrm{n}=544)$ & Mild $(\mathrm{n}=257)$ & Moderate $(\mathrm{n}=73)$ & Severe $(\mathrm{n}=29)$ & $p$ value \\
\hline In-hospital mortality & $48(8.8)$ & $25(9.7)$ & $5(6.8)$ & $5(17.2)$ & 0.406 \\
28-day mortality & $43(7.9)$ & $17(6.6)$ & $4(5.5)$ & $2(6.9)$ & 0.841 \\
ICU mortality & $29(5.3)$ & $14(5.4)$ & $3(4.1)$ & $2(6.9)$ & 0.948 \\
ICU length of stay (hour) & $43.4 \pm 201.9$ & $86.0 \pm 297.4$ & $49.9 \pm 31.7$ & $49.8 \pm 36.7$ & 0.092 \\
Hospital length of stay (day) & $141.8 \pm 89.3$ & $268.6 \pm 317.5$ & $295.7 \pm 215.4$ & $370.5 \pm 261.3$ & $<0.001$
\end{tabular}

Data are presented as numbers (\%) or means \pm standard deviations. ICU: intensive care unit.

Table 3. Multivariable analysis of in-hospital mortality according to the severity of hyponatremia

\begin{tabular}{lcc}
\hline Variable & Adjusted odds ratio $(95 \% \mathrm{CI})^{*}$ & $p$ value \\
\hline Hyponatremia & & 1 \\
$\quad$ No hyponatremia & $0.92(0.45-1.85)$ & Reference \\
Mild hyponatremia & $0.54(0.12-1.87)$ & 0.818 \\
$\quad$ Moderate hyponatremia & $5.41(1.23-20.54)$ & 0.368 \\
$\quad$ Severe hyponatremia & $3.45(1.29-9.72)$ & 0.017 \\
Malignancy & $6.15(1.65-23.38)$ & 0.016 \\
Continuous renal replacement therapy & $0.39(0.17-0.83)$ & 0.007 \\
ICP monitoring & $0.60(0.52-0.69)$ & 0.019 \\
Glasgow Coma Scale on ICU admission & $<0.001$ &
\end{tabular}

CI: confidence interval, APACHE: Acute Physiology and Chronic Health Evaluation, ICP: intracranial pressure, ICU: intensive care unit.

*Adjusted for hyponatremia, age, sex, comorbidities, cause of ICU admission, utilization of organ support modalities, use of invasive ICP monitoring device, hyperosmolar therapy, and APACHE II score. 


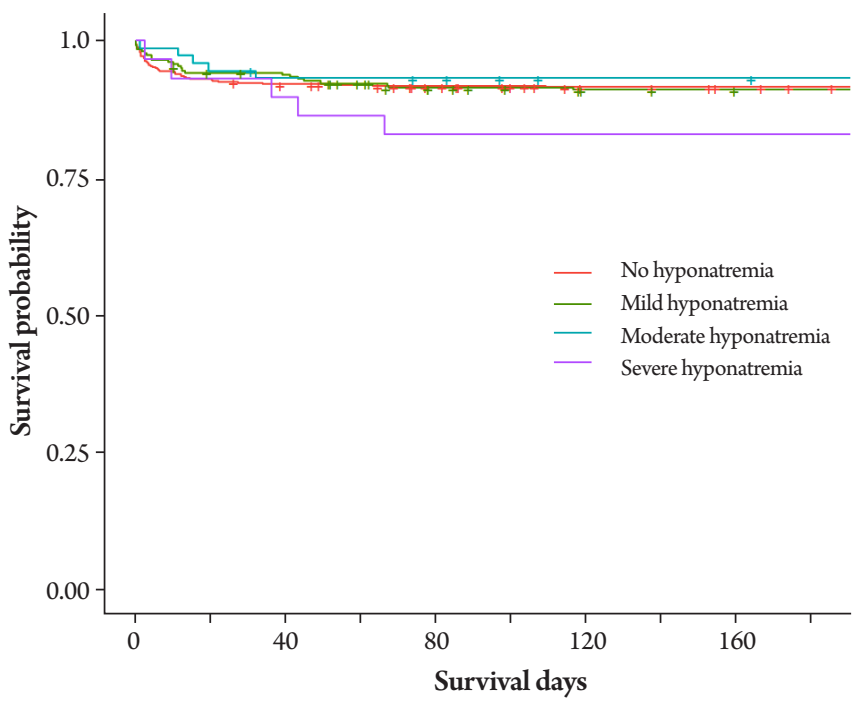

Fig. 2. Kaplan Meier curves of in-hospital mortality according to hyponatremia severity.

tracranial pressure, neurological abnormalities, and even death ${ }^{4,9)}$. Some complications, such as hyponatremic seizures, are more prevalent in severe cases of hyponatremia than in benign disease ${ }^{9)}$. For instance, the clinical manifestations of hyponatremia are severe in neurocritically ill patients with intractable intracranial hypertension, severe brain damage, or neurosurgical intervention ${ }^{6,16)}$.

Similar to the previous reports, in this study, hyponatremia was also a common electrolyte abnormality in neurocritically ill patients. However, most hyponatremia cases were mild and only 3\% of all patients showed severe hyponatremia. In the present study, severe hyponatremia was a significant predictor of in-hospital mortality, while mild to moderate hyponatremia were not associated with clinical outcomes. However, these findings are inconsistent with previous studies ${ }^{6,16)}$. Even patients with normal sodium level or hyponatremia were excluded from this study if they had a history of hypernatremia during ICU hospitalization. Several studies revealed that hypernatremia was associated with poor clinical outcomes in neurocritically ill patients ${ }^{8,10,11,18)}$. Although hyperosmolar therapy is the standard approach to the treatment of intracranial hypertension in the neurocritically ill patients, it is associated with hypernatremia and hyperosmolar therapy-induced acute kidney injury that can aggravate hypernatremia ${ }^{1,11,18)}$. In addition, hypothalamic dysfunction due to brain injury can contribute to hypernatremia $^{12,18)}$. Therefore, ICU-acquired hypernatremia is a possible complication in severe brain injuries. For this study, it is possible that some patients with severe brain injuries were excluded due to accompanied hypernatremia.

This study has several limitations. First, this was a retrospective review of medical records using data extracted from a Clinical Data Warehouse. The nonrandomized nature of registry data might have resulted in a selection bias. Second, the duration of hyponatremia and correction of serum sodium was not reflected in this study. Third, most hyponatremia cases were mild with only a small percentage of severe hyponatremia cases. Finally, the distribution of neurosurgical diseases was different from that in the general neurosurgical ICU, and the proportion of patients with brain tumors was particularly high.

\section{CONCLUSIONS}

Based on our study findings mild to moderate hyponatremia were not associated with clinical outcomes in neurocritically ill patients. However, severe hyponatremia was significantly associated with in-hospital mortality. Therefore, adequate and timely regulation of serum sodium level is important for favorable prognosis and survival of neurocritically ill patients.

\section{NOTES}

\section{Conflict of Interest}

No potential conflict of interest relevant to this article was reported.

\section{Informed Consent}

The requirement for informed consent was waived due to the retrospective nature of this study.

\section{Acknowledgements}

We would like to thank Hye Jung Kim, the nursing director of the neurosurgical intensive care unit, for providing excellent advice and engaging in fruitful discussions. We would also like to thank all nurses of the neurosurgery intensive care unit at Samsung Medical Center.

\section{REFERENCES}

1. Aiyagari V, Deibert E, Diringer MN. Hypernatremia in the neurologic intensive care unit: how high is too high? J Crit Care 2006;21:163-172.

2. Bennani SL, Abouqal R, Zeggwagh AA, Madani N, Abidi K, Zekraoui A, et al. [Incidence, causes and prognostic factors of hyponatremia in intensive care]. Rev Med Interne 2003;24: 224-229.

3. Boscoe A, Paramore C, Verbalis JG. Cost of illness of hyponatremia in the United States. Cost Eff Resour Alloc 2006;4:10. 
4. DeVita MV, Gardenswartz MH, Konecky A, Zabetakis PM. Incidence and etiology of hyponatremia in an intensive care unit. Clin Nephrol 1990;34:163-166.

5. Fogarty J, Loughrey C. Hyponatraemia in Hospitalised Adults: a Guide for the Junior Doctor. Ulster Med J 2017;86:84-89.

6. Hannon MJ, Thompson CJ. Neurosurgical Hyponatremia. J Clin Med 2014;3:1084-1104.

7. Hoorn EJ, Lindemans J, Zietse R. Development of severe hyponatraemia in hospitalized patients: treatment-related risk factors and inadequate management. Nephrol Dial Transplant 2006;21:70-76.

8. Hu B, Han Q, Mengke N, He K, Zhang Y, Nie Z, et al. Prognostic value of ICU-acquired hypernatremia in patients with neurological dysfunction. Medicine (Baltimore) 2016;95:e3840.

9. Kirkman MA, Albert AF, Ibrahim A, Doberenz D. Hyponatremia and brain injury: historical and contemporary perspectives. Neurocrit Care 2013;18:406-416.

10. Kolmodin L, Sekhon MS, Henderson WR, Turgeon AF, Griesdale DE. Hypernatremia in patients with severe traumatic brain injury: a systematic review. Ann Intensive Care 2013;3:35.

11. Li M, Hu YH, Chen G. Hypernatremia severity and the risk of death after traumatic brain injury. Injury 2013;44:1213-1218.

12. Maggiore U, Picetti E, Antonucci E, Parenti E, Regolisti G, Mergoni $\mathrm{M}$, et al. The relation between the incidence of hypernatre- mia and mortality in patients with severe traumatic brain injury. Crit Care 2009; 13:R110.

13. Manzanares W, Aramendi I, Langlois PL, Biestro A. Hyponatremia in the neurocritical care patient: An approach based on current evidence. Med Intensiva 2015;39:234-243.

14. Padhi R, Panda BN, Jagati S, Patra SC. Hyponatremia in critically ill patients. Indian J Crit Care Med 2014;18:83-87.

15. Qureshi AI, Suri MF, Sung GY, Straw RN, Yahia AM, Saad M, et al. Prognostic significance of hypernatremia and hyponatremia among patients with aneurysmal subarachnoid hemorrhage. Neurosurgery 2002;50:749-755; discussion 755-746.

16. Sherlock M, O'Sullivan E, Agha A, Behan LA, Rawluk D, Brennan $\mathrm{P}$, et al. The incidence and pathophysiology of hyponatraemia after subarachnoid haemorrhage. Clin Endocrinol (Oxf) 2006;64:250-254.

17. Vachharajani TJ, Zaman F, Abreo KD. Hyponatremia in critically ill patients. J Intensive Care Med 2003;18:3-8.

18. Vedantam A, Robertson CS, Gopinath SP. Morbidity and mortality associated with hypernatremia in patients with severe traumatic brain injury. Neurosurg Focus 2017;43:E2 .

19. Zada G, Liu CY, Fishback D, Singer PA, Weiss MH. Recognition and management of delayed hyponatremia following transsphenoidal pituitary surgery. J Neurosurg 2007;106:66-71. 